\section{International Scientific Journal Theoretical \& Applied Science}

\author{
p-ISSN: 2308-4944 (print) e-ISSN: 2409-0085 (online) \\ Year: $2016 \quad$ Issue: 9 Volume: 41 \\ Published: $30.09 .2016 \quad$ http://T-Science.org
}

SECTION 4. Computer science, computer engineering and automation.
Yuriy Konstantinovich Dimitriev

Associate Professor

Advanced Doctor in Engineering Sci., Rzhanov Institute of Semiconductor Phisics, Siberian Branch RAS, Russia dimi@isp.nsc.ru

Anatoliy Filippovich Zadorozhny $\mathrm{PhD}$ of Engineering Sci., Professor Novosibirsk State University of Architecture and Civil Engineering (Sibstrin), Russia 3af@ngs.ru

\title{
ON CONDITIONS OF LOCAL SELF-DIAGNOSIS OF MULTIPROCESSOR COMPUTING SYSTEMS UNDER MULTIPLE FAULTS
}

\begin{abstract}
Multiprocessor computing systems with point-to-point communication links in which t-multiple faults are allowed at the level of the processing modules are considered. For the known model of Preparata, Metze and Chien the conditions are found under which a technical state of each module can be determined by results of the comparative analysis of the outputs of tests relating to modules from its limited neighborhood. The state of the modules is defined in the course of diagnosis and its results are used for the management of sequence further testing of the system modules.

Key words: the multiprocessor computing systems, system diagnostics, graph models, local self-diagnosis, conditions of self-determination of a state of processor modules.

Language: Russian

Citation: Dimitriev YK, Zadorozhny AF (2016) ON CONDITIONS OF LOCAL SELF-DIAGNOSIS OF MULTIPROCESSOR COMPUTING SYSTEMS UNDER MULTIPLE FAULTS. ISJ Theoretical \& Applied Science, 09 (41): 52-61.

Soi: http://s-o-i.org/1.1/TAS-09-41-8 Doi: crossef http://dx.doi.org/10.15863/TAS.2016.09.41.8

\section{УДК 681.3.012+681.3-192}

\section{ОБ УСЛОВИЯХ ЛОКАЛЬНОГО САМОДИАГНОСТИРОВАНИЯ МНОГОПРОЦЕССОРНЫХ ВЫЧИСЛИТЕЛЬНЫХ СИСТЕМ ПРИ МНОЖЕСТВЕННЫХ ОТКАЗАХ}

Аннотация: Рассматриваются мультипроцессорные вычислительные системы со связями точкаточка, в которых допускаются t-кратные отказы на уровне обрабатывающих модулей. Для известной модели Препараты, Метияа и Чжена найдены условия, при которых техническое состояние каждого модуля может быть определено по результатам сопоставительного анализа исходов тестирования, относящихся к модулям из его ограниченной окрестности. Состояние модулей определяется в процессе диагностирования и его результаты используются для управления последовательностью дальнейшего тестирования модулей системы.

Ключевые слова: многопроцессорные вычислительные системы, системная диагностика, теоретикографовые модели, локальное самодиагностирование, условия самоопределения состояния процессорных модулей.
\end{abstract}

Работа выполнена при поддержке Российского фонда фундаментальных исследований (проект № 14-07-00169.а)

\section{1- Введение}

При конструировании масштабируемых многопроцессорных вычислительных систем (BC) в виде совокупности процессорных модулей, функциональные характеристики которых позволяют им в одиночку проверять и оценивать техническое состояние других модулей, их диагностические способности рассматриваются на системном уровне, т.е. на уровне отдельных обрабатывающих модулей и связей между ними. 
Рост сложности практически применяемых ВC опережает рост надежности обрабатывающих модулей, из которых система строится. Определяющей характеристикой ВС становится не ее производительность, а способность продолжать функционировать в течение продолжительного времени при наличии отказов ее элементов (живучее функционирование). Это ставит задачу автоматизировать процедуру определения технического состояния системы (системы с самодиагностированием). Для исследования диагностических свойств ВС на системном уровне используется теоретикографовая модель, предложенная в [1] и называемая ПМЧ-моделью. В этой модели идентификация технического состояния ВС осуществляется с помощью алгоритмов сопоставительного анализа исходов тестирования, выполняемого одними модулями системы над другими. При анализе учитывается диагностическая структура ВС — отношения тестируемый-тестирующий между ее модулями.

Мерой отказоустойчивости самодиагностируемых ВС служит кратность неисправностей $t$, представляющая собой наибольшее число одновременно присутствующих в системе неисправных модулей, при котором еще возможно определение ее состояния. Наличие в системе кратных неисправностей вызывает утрату тестами свойства надежности. Последнее означает, что оценки, получаемые исправными тестирующими модулями, соответствуют фактическому состоянию тестируемого модуля, а оценки, получаемые неисправными тестирующими модулями, не зависят от состояния тестируемого модуля.

Общим для многочисленных методов самодиагностики, известных из литературы (см., например, [2]), является то, что состояние ВС выводится из априорных предположений о техническом состоянии (исправный или неисправный) некоторых модулей, в общем случае выбираемых произвольно, и базируется на необходимости выполнения над модулями системы всех возможных тестов - независимо от фактического состояния системы. Самодиагностирование представляет собой процесс проб и ошибок, направленный на «вычисление» состояния ВС, которое не противоречит ни одному из полученных исходов тестирования при заданных правилах ненадежной интерпретации результатов тестов, выполняемых неисправными тестирующими модулями.

Основные недостатки описанного подхода состоят в следующем. 1. Процедура сбора информации для определения состояния ВС и «отката» при обнаружении ошибочности априорных предположений о состоянии выбранных модулей может захватывать всю систему. 2. Самодиагностика строится на базе сопоставительного анализа результатов полного множества тестов над системой независимо от фактического состояния ВС. 3. Целью самодиагностирования провозглашается обнаружение неисправных модулей системы. Идентификация исправных модулей рассматривается как побочный результат.

Эти недостатки ограничивают возможность применения подхода [1] к самодиагностике живучих систем. Для таких систем перспективна постановка задачи системного диагностирования, как проблемы идентификации хотя бы одного исправного модуля. Выделенные один или группа исправных модулей составляют диагностическое ядро системы. Модули ядра используются для определения состояния ещё не проверенных модулей, в том числе - идентификации новых исправных модулей, пополняющих диагностическое ядро. Тем самым реализуется известный ещё для компьютеров первых поколений принцип диагностирования методом расширяющегося диагностического ядра. При использовании диагностического ядра состояние любого модуля, смежного с модулем из ядра, определяется по исходу единственного теста над ним. Это уменьшает число тестов, необходимых для определения состояния системы и, как следствие, увеличивает время использования системы по назначению. Использование метода выделения диагностического ядра обеспечивает бОльшую надежность защиты системы от разрушительного воздействия со стороны еще не выявленных неисправных модулей и бОльшую оперативность реакции на попытку такого воздействия. Образуемая на базе ядра связанная подсистема из исправных модулей может немедленно переключаться на решение прикладных задач, что создаёт основу для совмещения счета с диагностикой и организации живучего функционирования ВС. Впервые использование метода выделения диагностического ядра для самодиагностирования описано в [3].

В [4, 5] для модели ПМЧ предложен «безоткатный» метод самодиагностики с помощью функционально выделяемого диагностического ядра для случая кратных устойчивых отказов. Метод допускает использование адаптивных децентрализованных алгоритмов, позволяющих достичь для системы свойства живучести. Как выделение диагностического ядра, так и идентификация состояния остальных модулей системы осуществляются на основе анализа исходов тестирования, получаемых в ходе тестирования системы. Идентификация состояния модулей ВС осуществляется по результатам тестирования 
модулей, находящихся в их ограниченной окрестности, в связи с чем предложенный метод назван методом локального самодиагностирования. В [4, 5] введено определение и доказаны необходимые и достаточные условия диагностируемости при использовании ограниченного набора локальных признаков идентификации состояния модулей, названного базовыми условиями самоопределения. Отличительной особенностью базовых условий самоопределения является то, что они основаны на анализе лишь тех исходов тестирования, которые позволяют идентифицировать действительное состояние модуля.

В $[5,6]$ найдены новые признаки локального самоопределения, использование которых ведет к построению более эффективных децентрализованных адаптивных алгоритмов самодиагностирования.

В данной статье проводится систематизация известных и предлагаются новые условия самоопределения, обеспечивающие построение эффективных децентрализованных адаптивных алгоритмов самодиагностирования для живучих ВС. Новизна используемого подхода состоит в возможности корректировать значение проектной характеристики самодиагностики ВC кратности неисправностей - по числу неисправных модулей, достоверно выявленных в ходе самодиагностирования, что ведёт к уменьшению числа тестов, необходимых для определения состояния системы в целом.

\section{2- Определения и обозначения}

Диагностическая структура ВС представлена ориентированным графом $D=(V, E) \quad$ с взвешенными дугами, в котором $N=|V|$; здесь $|X|$ означает мощность множества $X$. Вершины $v \in V$ графа отображают модули, а дуги $(v, w) \in E, \quad v, w \in V$, - тестовые связи между ними. Вес $a(v, w)$ дуги $(v, w)$ диагностического графа равен двоичному исходу соответствующего теста: $a(v, w)=0$, если модуль $v$ считает, что модуль $w$ исправен, и $a(v, w)=1$ в противном случае. Как сказано выше, для ПМЧ-модели исход теста, выполняемого исправным модулем, достоверен, а исход теста, выполняемого неисправным модулем ненадежен: он не зависит от фактического состояния тестируемого.

Чтобы упростить изложение, переносим терминологию системы на представляющий ее граф.

Состояние графа описываем перечислением присутствующих в графе неисправных вершин
$F_{k}=\left\{v_{i}\right\} \quad$ и называем его «образом неисправностей». Множество $F(t)=\left\{F_{k}\right\}$ допустимых неисправностей составляют все возможные сочетания из $N$ вершин по $n$, где $n=\overline{1, t}$, а $t$-кратность неисправностей. Рассматриваются отказы - устойчивые неисправности модулей ВС. Упорядоченное множество $\sigma\left(F_{k}\right)$ исходов тестов, которое может быть получено для заданного образа неисправностей $F_{k}$, называем синдромом состояния графа. Синдром $\sigma\left(F_{k}\right)$ совместен с образом неисправностей $F_{k}$, а $F_{k}$ порождает синдром $\sigma\left(F_{k}\right)$.

Для модуля, которому сопоставлена вершина $v, \quad$ множества $\Gamma^{-1}(v)=\{w \in V: \quad(w, v) \in E\}$,

$\Gamma(v)=\{w \in V: \quad(v, w) \in E\}$ и $H(v)=\Gamma^{-1}(v) \cup \Gamma(v)$ представляют в диагностическом графе модули тестирующие, тестируемые и смежные. Согласно весу дуг, инцидентных вершине $v$, выполняется: $\Gamma^{-1}(v)=\Gamma_{0}^{-1}(v) \cup \Gamma_{1}(v), \quad \Gamma(v)=\Gamma_{0}(v) \cup \Gamma_{1}(v)$. Здесь $\Gamma_{0}^{-1}(v) \cap \Gamma_{1}(v)=\varnothing$ и $\Gamma_{0}(v) \cup \Gamma_{1}(v)=\varnothing$.

Граф называем $t$-диагностируемым, если и только если по заданному синдрому можно идентифицировать все неисправные вершины диагностического графа, когда их число не превышает заданного значения $t$ кратности неисправностей. В [7] показано, что если в графе нет взаимно тестируемых вершин, то необходимыми и достаточными условиями $t$ диагностируемости являются: 1) $N \geq 2 t+1$; 2) $\forall v \in V\left\{\left|\Gamma^{-1}(v)\right| \geq t\right\}$. Далее рассматриваем графы, удовлетворяющие этим условиям.

\section{3- Алгоритм самодиагностирования}

В [4-6, 9-10] описан подход к диагностированию живучих $\mathrm{BC}$, основанный на том, что фактическое состояние каждой вершины $v, \quad v \in V$, диагностического графа $D=(V, E)$ определяется с помощью правил сопоставительного анализа исходов тестирования, которые относятся только к вершинам из ее ограниченной (и способной к росту в ходе диагностирования) окрестности и называются правилами (локального) самоопределения.

Алгоритм локального самодиагностирования представляет собой итеративный процесс и состоит из чередующихся шагов 1) тестирования, завершающегося определением значений элементов синдрома, которые относятся к участвующим в 
тестировании вершинам, и 2) сопоставительного анализа полученного фрагмента синдрома. Результатом сопоставительного анализа является изменение состояния вершин диагностического графа (или просто "состояния графа"). Состояние графа описывается с помощью меток, сопоставленных его вершинам. Каждой вершине $v$ сопоставлена метка $m(v) \in M$, $M=\{0,1,2,3, \ldots\}^{1}$. Значение $m(v)=2$, если фактическое состояние вершины $v$ не идентифицировано (в частности, перед началом диагностирования); значение $m(v)=0$ или $m(v)=1$, если по результатам анализа текущего значения синдрома вершина $v$ признана соответственно исправной или неисправной (эти состояния называем финальнылм). Совокупность найденных финальных меток вершин, так же, как сам процесс их определения, называем разметкой графа. Разметка графа завершается, когда установлено финальное состояние для всех его вершин ${ }^{2}$.

При выполнении разметки поведение каждой вершины графа зависит от состояния смежных с нею вершин. Каждой вершине $v$ графа присваиваются метки-списки, сопоставленные некоторым выделенным состояниям из множества $M$. В процессе разметки вершины обмениваются меткамисписками, так что каждая из них знает состояние вершин из некоторой своей окрестности. Величина и состав окрестности для каждой вершины индивидуален и зависит от порядка тестирования, исходов выполненных тестов, от задержек в пересылке индивидуальных метоксписков и др. Важно, что каждая вершина "вычисляет" свое состояние, исходя из текущего значения ее меток-списков.

\section{3. Базовые условия локального самоопределения}

Разметка диагностического графа осуществляется по шагам. Если на очередном $r$-м шаге идентифицирована неисправная вершина $v$, то в дальнейшем условия самоопределения для смежных с нею вершин $w \in H(v)$

${ }^{1}$ Мощность множества $M$ зависит от выбора условий самоопределения, используемых в конкретном алгоритме самодиагностирования.

2 Термин разметка графа применяем как к процессу определения финального состояния вершин графа, так и к описанию множества вершин графа, получивших метки финального состояния на некотором шаге самодиагностирования. рассматриваются на остаточном множестве $R^{(r)}(w)$, образованном исключением из $H(w)$ вершины $v$ (равно как и других смежных с нею идентифицированных неисправных вершин).

Существенной новой особенностью развиваемого подхода к локальному самодиагностированию является возможность оперативной корректировки значения проектной характеристики системы - кратности допустимых неисправностей $t-$ в случае, когда для некоторых вершин устанавливается финальное состояние неисправности ${ }^{3}$. Установка на шаге $r$ самодиагностирования финального состояния неисправности для множества $f^{(r)}(v)$, $\left|f^{(r)}(v)\right|=\tau^{(r)}(v), \quad$ вершин из окрестности, учитываемой в вершине $v$, приводит к корректировке еe индивидуального порога самоопределения

$Q^{(r)}(v)$ : $Q^{(r)}(v):=Q^{(r-1)}(v)-\tau^{(r)}(v)$. Будем называть $\tau^{(r)}(v)$ величиной корректировки порога самоопределения вершины $v$ на_ $r$-м шаге самодиагностирования.

Начальное состояние графа задается следующими значениями, указываемыми для каждой вершины графа:

$$
\left\{\begin{array}{c}
R^{(0)}(v)=H(v) \\
\tau^{(0)}(v)=0 \\
f^{(0)}(v)=\varnothing \\
Q^{(r)}(v)=t \\
m^{(0)}(v)=2
\end{array}\right.
$$

Начальное

$$
\text { значение }
$$

порога самоопределения для всех вершин графа равно величине кратности неисправностей $t$, поскольку априорная информация о мощности образа неисправностей отсутствует. Порог меняется по мере определения финального состояния для новых вершин.

На $r$-м шаге разметки диагностического графа, $r=1,2, \ldots$, для каждой вершины графа выполняются следующие преобразования:

$$
\begin{aligned}
& {\left[\left|R^{(r-1)}\left(\Gamma_{1}^{-1}(v)\right) \cup R^{(r-1)}\left(\Gamma_{1}(v)\right)\right|>\right.} \\
& \left.>\left(Q^{(r-1)}(v)-\tau^{(r-1)}(v)\right)\right] \mapsto m^{(r)}(v):=1, \\
& {\left[\left|R^{(r-1)}\left(\Gamma_{0}^{-1}(v)\right)\right| \geq\left(Q^{(r-1)}(v)-\tau^{(r-1)}(v)\right)\right] \mapsto} \\
& \mapsto m^{(r)}(v):=0
\end{aligned}
$$

3 Чтобы подчеркнуть первичность влияния соглашения о кратности допустимых неисправностей на диагностические свойства системы, его называют аксиомой кратности. 


$$
\begin{aligned}
& m^{(r)}(v):=0 \mapsto\left[\forall w \in \Gamma_{0}(v)\left\{m^{(r)}(w):=0\right\}\right] \& \\
& \&\left[\forall w \in\left(\Gamma_{1}^{-1}(v) \cup \Gamma_{1}(v)\right)\left\{m^{(r)}(w):=1\right\}\right] \\
& \left.m^{(r)}(v):=1 \mapsto \forall w \in \Gamma_{0}^{-1}(v)\left\{m^{(r)}(w):=1\right\}\right], \\
& m^{(r)}(v):=0 \rightarrow\left[T(v):=\Gamma_{1}^{-1}(v) \cup \Gamma_{1}(v) ;\right. \\
& \forall w \in\left(H(v)-\Gamma_{1}^{-1}(v)-\Gamma_{1}(v)-\Gamma_{0}(v)\right)\{f(w):=, \\
& \left.\left.:=f(w) \cup T(v) ; \quad \tau^{(r)}(w):=|f(w)|\right\}\right] \\
& m^{(r)}(v):=1 \rightarrow\left[T(v):=\Gamma_{0}^{-1}(v) \cup v ;\right. \\
& \forall w \in\left(H(v)-\Gamma_{0}^{-1}(v)\right)\{f(w):=f(w) \cup T(v) ; \\
& \left.\left.\tau^{(r)}(w):=|f(w)|\right\}\right] \\
& \left\{\begin{array}{l}
R^{(r)}(w)=H(w)-\left\{v \cup \Gamma_{0}^{-1}(v)\right], \text { если } m(v)=1, \\
R^{(r)}(w)=H(w)-\left\{\Gamma_{1}^{-1}(v) \cup \Gamma_{1}(v) \cup\right. \\
\left.\left.\cup\left\{v \cup \Gamma_{0}(v)\right\}\right\}\right], \text { если } m(v):=0 .
\end{array}\right.
\end{aligned}
$$

Здесь $\quad m(v):=z \quad$ обозначает операцию присвоения вершине $v$ метки финального состояния: $z \in\{0,1\}$. Выражения (1) и (2) указывают базовые прямые условия самоопределения - признаки финального состояния вершины, исходя из значений синдрома, относящихся только к ней самой. Выражения (3) и (4) представляют базовые выводимые условия самоопределения условия установки финального состояния смежных вершин, являющейся следствием идентификации финального состояния вершины $v$ согласно (1) и (2). Преобразования в выражениях (3)-(4) вытекают из правил определения исходов тестирования для ПМЧ-модели, в соответствии с которыми

$\left[\Gamma_{0}(v) \subset\left(V-F_{k}\right) \&\left[\Gamma_{1}^{-1}(v) \cup \Gamma_{1}(v)\right] \subseteq F_{k}\right], \quad$ если вершина $v$ исправна, и $\Gamma_{0}^{-1}(v) \subset F_{k} X_{0}(i) \subset F_{k}$, если вершина $v$ неисправна. В выражениях (5) и (6), задающих условия корректировки порога самоопределения для вершины $v, T(v)$ и $f(w)-$ списки, сопоставляемые вершинам $v$ и $w$ соответственно. Выражения (7) определяют остаточные множества для вершин, не перешедших на $r$-м шаге в финальное состояние.

\section{4- Функции совместности}

В [11] для описания свойств используемых ненадежных тестов предложено использовать булевы функции, называемые элементарными функциями совместности. Элементарная функция совместности перечисляет состояния пары вершин, участвующих в выполнении теста $(v, w)$ , которые соответствуют заданному исходу $z \in\{0,1\}$ этого теста. Для ПМЧ-модели элементарные функции совместности имеет следующий вид.

$$
\begin{aligned}
f_{0}(v, w) & =\bar{v} \bar{w} \vee \bar{v} w \vee v w, \\
f_{1}(v, w) & =\bar{v} \bar{w} \vee \bar{v} w \vee v \bar{w} .
\end{aligned}
$$

Пусть $F_{k}-$ образ неисправностей диагностического графа $D, \quad \sigma^{(r)}\left(F_{k}\right) \quad-$ фрагмент порождаемого им синдрома, сформированный на $r$ шагах разметки, $\sigma^{(r)}\left(F_{k}\right) \subset \sigma\left(F_{k}\right), \quad$ и $\quad D_{T} \quad-\quad$ подграф диагностического графа (называемый далее подграфом тестирования), индуцируемый элементами $\sigma^{(r)}\left(F_{k}\right)$.

Определение 1. Для фрагмента синдрома $\sigma^{(r)}\left(F_{k}\right)$ функичией совместности называется конъюнкиия элементарных функиий z-совместности, соответствующих значениям элементов из $\sigma^{(r)}\left(F_{k}\right)$, приведенная $\kappa$ совершенной дизъюнктивной нормальной форме (СДНФ) и из которой исключень минтермы, содержащче более $Q^{(r)}\left(D_{T}\right)$ переменных в инверсной форме. Здесь $Q^{(r)}\left(D_{T}\right)$ - порог самоопределения, рассчитанный на $r$-м шаге разметки для подграфа $D_{T}$.

Определение 2. Функция совместности называется определяющей, если она имеет хотя бы одну общую переменную в прямой или инверсной форме. Выделенные общие переменные называем определимыми.

Определение 3. Функиия совместности называется продуктивной, если ее форма позволяет уточнить (или ограничить) область размещения вершин определенного состояния, их число или взаимное расположение.

Функции совместности, удовлетворяющие определениям 2 или 3, называем значимыми. Наличие или отсутствие свойства значимости заданной функции совместности ассоциируем с неформальным понятием "диагностические свойства" 4 соответствующего подграфа тестирования.

\section{5- Условия группового самоопределения}

Функция 0-совместности (8) задает отношение частичного порядка на множестве

4 Под диагностическими свойствами понимаем любые количественные или качественные показатели, которые позволяют оценить достижимость или эффективность самодиагностирования, а также признаки, которые позволяют идентифицировать состояние отдельных модулей системы. 


\begin{tabular}{|c|c|c|c|c|c|c|}
\hline Impact Factor: & $\begin{array}{l}\text { ISRA (India) } \\
\text { ISI (Dubai, UAE } \\
\text { GIF (Australia) } \\
\text { JIF }\end{array}$ & $\begin{array}{r}=1.344 \\
=0.829 \\
=0.564 \\
=1.500\end{array}$ & $\begin{array}{l}\text { SIS (USA) } \\
\text { PИНЦ (Russia) } \\
\text { ESJI (KZ) } \\
\text { SJIF (Morocco) }\end{array}$ & $\begin{array}{l}=0.912 \\
=0.234 \\
=1.042 \\
=\mathbf{2 . 0 3 1}\end{array}$ & $\begin{array}{l}\text { ICV (Poland) } \\
\text { PIF (India) } \\
\text { IBI (India) }\end{array}$ & $\begin{array}{l}=6.630 \\
=1.940 \\
=4.260\end{array}$ \\
\hline
\end{tabular}

вершин, участвующих в выполнении теста $(v, w)$ , по их состоянию: метка состояния тестируемой вершины не больше метки тестирующей. Транзитивность отношения (8) на содержательном уровне означает, что в простом 0-пути неисправные вершины предшествуют исправным.

Пример. Рассмотрим фрагмент синдрома

$$
\begin{aligned}
& \sigma^{(r)}\left(P_{0}\left(v_{0}, v_{3}\right)\right)=\left\{a\left(v_{0}, v_{1}\right)=0,\right. \\
& \left.a\left(v_{1}, v_{2}\right)=0, a\left(v_{2}, v_{3}\right)=0\right\}
\end{aligned}
$$

который соответствует подграфу тестирования, представляющего простой путь из вершины $v_{0}$ в вершину $v_{3}$. Для него функция совместности имеет вид:

$$
\begin{aligned}
& P_{0}\left(v_{0}, v_{3}\right)=f_{0}\left(v_{0}, v_{1}\right) f_{0}\left(v_{1}, v_{2}\right) f_{0}\left(v_{2}, v_{3}\right)= \\
& =\left(\overline{v_{0}} \vee v_{1}\right)\left(\overline{v_{1}} \vee v_{2}\right)\left(\overline{v_{2}} \vee v_{3}\right)= \\
& =\overline{v_{0}} \overline{v_{1}} \overline{v_{2}} \overline{v_{3}} \vee \overline{v_{0}} \overline{v_{1}} \overline{v_{2}} v_{3} \vee \overline{v_{0}} \overline{v_{1}} v_{2} v_{3} \vee \overline{v_{0}} v_{1} v_{2} v_{3} \vee v_{0} v_{1} v_{2} v_{3} .
\end{aligned}
$$

Для простого контура, порождающего синдром $\sigma\left(\left[P_{0}\left(v_{0}, v_{3}\right)\right] f_{0}\left(v_{3}, v_{0}\right)\right)$, отношение транзитивности приводит к эквивалентности финального состояния его вершин:

$$
P_{0}\left(v_{0}, v_{3}\right) f_{0}\left(v_{0}, v_{1}\right)=\overline{v_{0}} \overline{v_{1}} \overline{v_{2}} \overline{v_{3}} \vee v_{0} v_{1} v_{2} v_{3} .
$$

$\mathrm{He}$ останавливаясь на других конструкциях связных подграфов тестирования, описываемых функцией совместности вида (10), сформулируем их общее свойство.

Св о йство 1 (условие группового самоопределения). Если для связного 0-подграфа $D_{0}=\left(V_{0}, E_{0}\right)$, описьваемого функцией (10), найдется вершина $w, w \notin V_{0}$, смежная хотя бы с одной верииной $v_{k} \in V_{0}, u$ такая, что $m(w)=0$, то финальное состояние всех вершин подграфа $D_{0}$ устанавливается по исходу единственного mecma $\left(w, v_{k}\right)$.

Из свойства группового самоопределения вытекает следующее.

Св в йство 2. Если для связного подграфа $D_{0}=\left(V_{0}, E_{0}\right)$ функиия совместности имеет вид (10), то

1. Когда $\left|V_{0}\right| \geq Q^{(r)}\left(D_{0}\right)$, то все вершины из $V_{0}$ исправнь: $V_{0} \not \subset F \rightarrow \forall v_{k} \in V_{0}\left\{m\left(v_{k}\right):=0\right\}$;

2. Когда для некоторой вершины $v_{k} \in V_{0}$ устанавливается финальное состояние $m\left(v_{k}\right) \in\{0,1\}, \quad$ то для каждой вершинь $v_{i} \in\left\{V_{0}-v_{k}\right\}, v_{i} \neq v_{k}$ выполняется $m\left(v_{i}\right):=m\left(v_{k}\right)$.

Следующее свойство указывает условия объединения компонент 0-связности, описываемых функцией (10).

Св в йство 3. Если в подграфе тестирования существуют компоненть 0- связности $\quad D_{0}^{\prime}=\left(V_{0}^{\prime}, E_{0}^{\prime}\right) \quad u \quad D_{0}^{\prime \prime}=\left(V_{0}^{\prime \prime}, E_{0}^{\prime \prime}\right)$, описываемые функиией (10) и такие, что $v \in V_{0}^{\prime}$, $w \in V_{0}^{\prime \prime}$, а вершины $v$ и $w$ смежны в графе $D$, то $\operatorname{nри} a(v, w)=a(w, v)=0$ верииньи из $D_{0}^{\prime}$ и из $D_{0}^{\prime \prime}$ имеют одинаковое состояние и эти компоненть могут быть объединены в одну.

\section{6- Треугольники тестирования}

Базовые условия (1), (2) самоопределения вершины $v$ учитывают исходы тестирования, выполняемого на открытой окрестности этой вершины, а именно, исходы тестов вида $(w, v)$ и $(v, w)$, где $w \in H(v)$. Рассмотрение замкнутой окрестности $v \cup H(v)$ дает новые условия самоопределения [8].

Пусть для вершины $v$ диагностического графа $\quad\{w, u\} \subset H(v) . \quad$ Рассмотрим ориентированный полный граф $K_{3}$ с множеством вершин $\{v, w, u\}$. Назовем треугольником тестирования ориентированный подграф графа $K_{3}$, не содержащий взаимно обратных дуг. Для вершины $v$ диагностического графа множество треугольников тестирования на множестве $\{v, w, u\}$ задается выбором дуг $K_{3}$. В $[6,8]$ выделены два основных треугольника тестирования, описываемых наборами дуг: $A_{1}=\{(v, w),(v, u),(w, u)\}$ $A_{2}=\{(v, w),(w, u),(u, v)\}$. Показано, что все остальные треугольники тестирования эквивалентны основным с точностью до обозначения вершин.

В [6] показано, что для треугольника тестирования $A_{1}$ синдромы $\sigma_{11}^{(r)}\left(A_{1}\right)=\{a(v, w)=0, a(v, u)=0, a(w, u)=1\}$ и $\sigma_{12}^{(r)}\left(A_{1}\right)=\{a(v, w)=0, a(v, u)=1, a(w, u)=0\}$ дают функцию совместности, которая составляет условие самоопределения неисправной вершины $v: m(v)=1$. В треугольнике тестирования вида $A_{2}$ синдромы

$$
\begin{aligned}
& \sigma_{21}^{(r)}\left(A_{2}\right)=\{a(v, w)=0, a(v, u)=0, a(w, u)=1\}, \\
& \sigma_{22}^{(r)}\left(A_{2}\right)=\{a(v, w)=0, a(v, u)=1, a(w, u)=0\} \\
& \sigma_{22}^{(r)}\left(A_{2}\right)=\{a(v, w)=1, a(v, u)=0, a(w, u)=0\}
\end{aligned}
$$

составляют соответственно условие самоопределения неисправности вершины $v$, вершины $w$ и вершины $u$.

Из всего множества треугольников тестирования, которые можно построить на множестве $V(v)=v \cup H(v), \quad$ выделены те, которые обеспечивают самоопределение вершины $\quad v$. Для вершин $\quad w, u \in H(v)$ 
идентификация финального состояния обеспечивается из аналогичных треугольников тестирования, построенных на множествах $V(w)=w \cup H(w) \quad$ и $\quad V(u)=u \cup H(u)$ соответственно.

Принципиальную

возможность эффективного использования треугольников тестирования показывает установленное в [8] свойство достаточности использования треугольников тестирования вида $A_{2}$ в сочетании с условиями (1) и (2) для достижения локальной $t$-диагностируемости для класса циркулянтных диагностических графов, описанных в [1].

\section{7- Обратное тестирование}

В практике построения многопроцессорных вычислительных систем со связями точка-точка определилась тенденция к использованию однородных вершинно симметричных рабочих графов $G=\left(V_{W}, E_{W}\right)$. Структура рабочего графа задана областью применения ВC. В вершинно симметричном графе все вершины эквивалентны по их положению. Это позволяет применять для управления системой децентрализованные отказоустойчивые алгоритмы, упрощает структуру операционной системы, в частности, облегчает построение операционной системы для масштабируемой ВС. Отмеченные свойства делают вершинно симметричные графы привлекательными также и для использования в живучих ВС.

По экономическим соображениям диагностический граф $D=(V, E)$ ВС реализуется как подграф рабочего графа $G=\left(V_{W}, E_{W}\right)$, так что $V=V_{W}$ и $E \subseteq E_{W}$. Рабочий граф имеет симметрические дуги: если в рабочем графе $G=\left(V_{W}, E_{W}\right) \quad$ выполняется $(v, w) \in E_{W}, \quad$ то и $(w, v)=E_{W}$. Классическая модель самодиагностирования [1] предполагает использование ориентированных диагностических графов с асимметричными дугами, что составляет необходимое и достаточное условие для достижения оптимальности диагностического графа. В оптимальном диагностическом графе число дуг при заданном $t$ минимально.

Однако применение оптимального диагностического графа в виде подграфа рабочего приводит к неполному использованию в целях самодиагностирования возможностей последнего. Другими словами, если вершины $v$ и $w$ смежны в рабочем графе, то из двух возможных между ними связей $(v, w)$ и $(w, v)$ рабочего графа для реализации диагностического подграфа используется лишь одна.
Предположим для определенности, что в диагностический граф входит дуга $(v, w)$. Назовем тест, реализуемый этой дугой, прямым. Рассмотрим возможность, которую предоставляет для самоопределения вершин графа использование противоположно направленной дуги рабочего графа, соответствующую тесту, называемому обратнылм.

Рассмотрим функции совместности для всех возможных значений синдрома $\sigma^{(r)}(v, w)=\{a(v, w), a(w, v)\}$

при $a(v, w), a(w, v) \in\{0,1\}$. Анализ этих синдромов дает следующие условия определимости вершин диагностического графа.

\section{Свойство 4.}

$$
\sigma^{(r)}(v, w)=\{a(v, w)=1, a(w, v)=0\} \mapsto m(w):=1
$$

т.е. вершина $w$ самоопределима как неисправная.

Свойство 5.

$$
\begin{aligned}
& \sigma^{(r)}(v, w)=\{a(v, w)=0, a(w, v)=0\} \mapsto \\
& \mapsto m(v)=m(w)
\end{aligned}
$$

т.е. финальное состояние вершин $v \quad u \quad w$ совпадает.

В [9] получены условия, при которых симметричный циркулянтный диагностический граф, описанный в [1], $t$-диагностируем при использовании обратного тестирования совместно с условиями базовой определимости (1) и (2).

\section{8- Условное самоопределение}

Симметрический характер функции 1совместности (9) позволяет рассматривать неориентированные 1-подграфы тестирования.

Пусть $D_{1}=\left(V_{1}, E_{1}\right) \quad$ - (связный) неориентированный 1-подграф тестирования. Для него функция 1-совместности $\Phi\left(D_{1}\right)=\bigcap_{\left(v_{i}, v_{j}\right) \in E_{1}}\left(\overline{v_{i}} \vee \overline{v_{j}}\right), \quad$ приведенная $\quad$ к СДНФ, перечисляет все его вершинные покрытия [12], а полученная из нее сокращенная дизъюнктивная форма указывает все его минимальные вершинные покрытия:

$$
\Phi\left(D_{1}\right)=\bigcap_{\left(v_{i}, v_{j}\right) \in E_{1}}\left(\overline{v_{i}} \vee \overline{v_{j}}\right)=\bigcup_{k=1}^{p} \mu_{k} .
$$

Здесь $\mu_{k}-$ терм, $\mu_{k} \subseteq V_{1}$, указывающий состав $k$-го по счету вершинного покрытия графа $D_{1}$, а $p$ - число минимальных покрытий. Терм минимального ранга соответствует наименьшему покрытию, а ранг этого терма есть число вершинного покрытия. Форма (11) единственная, поскольку литералы всех термов взяты в инверсной форме. Термы функции $\Phi\left(D_{1}\right)$ попарно различны и представляют собой еe простые импликанты, а сама функция $\Phi\left(D_{1}\right)-$ 


\begin{tabular}{|c|c|c|c|c|c|c|}
\hline Impact Factor: & $\begin{array}{l}\text { ISRA (India) } \\
\text { ISI (Dubai, UAE } \\
\text { GIF (Australia) } \\
\text { JIF }\end{array}$ & $\begin{array}{r}=1.344 \\
=0.829 \\
=0.564 \\
=1.500\end{array}$ & $\begin{array}{l}\text { SIS (USA) } \\
\text { PИНЦ (Russia) } \\
\text { ESJI (KZ) } \\
\text { SJIF (Morocco) }\end{array}$ & $\begin{array}{l}=0.912 \\
=0.234 \\
=1.042 \\
=\mathbf{2 . 0 3 1}\end{array}$ & $\begin{array}{l}\text { ICV (Poland) } \\
\text { PIF (India) } \\
\text { IBI (India) }\end{array}$ & $\begin{array}{l}=6.630 \\
=1.940 \\
=4.260\end{array}$ \\
\hline
\end{tabular}

тупиковая ДНФ, перечисляющая все минимальные покрытия заданного 1-подграфа неисправными вершинами.

Условимся, что термы в выражении (11) перенумерованы в порядке возрастания числа литералов в них, так что $\left|\mu_{1}\right| \leq\left|\mu_{2}\right| \leq \ldots \leq\left|\mu_{p}\right|$. В таком случае значение $\left|\mu_{1}\right|$ указывает число вершинного покрытия рассматриваемого 1подграфа.

Возможность самоопределения для 1подграфа общего вида $D_{1}$ основана на том, что при пороге самоопределения $q<\left|V_{1}\right|, \quad q=Q^{(\tau)}\left(D_{1}\right)$, функция (11) принимает вид $\Phi\left(D_{1}\right)=\bigcup_{k=1}^{p^{\prime}} \mu_{k}$, где $p^{\prime}$ наибольший ранг простых импликант функции (11) такой, что $p^{\prime} \leq q$. Поэтому диагностические свойства функции совместности для 1-подграфа общего вида определяются следующим.

Свойство 6. Если в 1-подграфе тестирования $D_{1}$ для заданного образа неисправности $F_{j}$ пи пороге самоопределения $q=Q^{(r)}\left(D_{1}\right)$, функичия $\Phi\left(D_{1}\right)=\bigcup_{k=1}^{p^{\prime}} \mu_{k}, p^{\prime} \leq q$, такова, что:

1) не имеет общих переменных, то эта функиия совместности непродуктивна;

2) имеет общие переменные $\overline{v_{1}}, \overline{v_{2}}, \ldots, \overline{v_{i}}$, то 1-подграф тестирования $D_{1}$ имеет самоопределяемые вершины: $v_{1}, v_{2}, \ldots, v_{i} \in F_{j}$;

3) состоит из единственного терма $\mu_{1}$, то все вершины 1-подграфа тестирования самоопределяемые, а именно: $\mu_{1} \subset F_{j}$ и $\left\{V_{1}-\mu_{1}\right\} \subseteq\left(V-F_{j}\right)$. При выполнении условий (1) или (2) осуществляется соответствуюшая корректировка порога самоопределения. В случае (3) идентифииируются исправные вершины, поэтому корректировка порога самоопределения не производится.

Подчеркнем, что в случае выполнения свойства 6.3 имеет место самоопределение для исправных вершин диагностического графа. Насколько известно авторам, определимость исправных вершин из рассмотрения 1-подграфов тестирования ранее не описывалась.

Итак, для идентификации финального состояния вершин для 1-подграфа тестирования общего вида на основе свойства 6 требуется решить задачу о перечислении его минимальных вершинных покрытий. Известно, что даже задача o построении некоторого (любого) минимального вершинного покрытия для заданного графа в общем случае является $N P$ полной. Это требует поиска приближенных решений задачи о вершинном покрытии для 1подграфов. Одно из таких решений состоит в следующем.

Продуктивность элементарной функции 1совместности (9) состоит в том, что единичный исход теста не идентифицирует неисправные вершины точно, а указывает на пересечение множества вершин, участвующих в тестировании, с заданным образом неисправностей, выделяя подмножество вершин, к которому они принадлежат, согласно следующему.

$$
\begin{aligned}
& \text { С в о й с т в о } 7 . \\
& \forall a(v, w)=1 \in \sigma\left(F_{k}\right)\left\{\{v, w\} \cap F_{k} \neq \varnothing\right\} . \\
& \text { Выполнение условия } \quad\{v, w\} \cap F_{k} \neq \varnothing
\end{aligned}
$$
означает, что вершины $\{v, w\}$ могут быть исключены из диагностического графа вместе с инцидентной им дугой, а текущее значение порога самоопределения уменьшено на единицу. Поэтому достигаемую определимость и соответствующую корректировку порога самоопределения называем условными.

$$
\text { При использовании условного }
$$
самоопределения из подграфа тестирования удаляются как неисправные, так и исправные вершины. Это требует контроля за соблюдением аксиомы кратности, согласно которой общее число исправных вершин в остаточном графе должно быть больше числа неисправных вершин, что следует из условия $N \geq 2 t+1$, характеризующего ПМЧ-модель самодиагностирования.

Свойство самоопределения 7 позволяет получать приближенное решение задачи о вершинном покрытии графа в виде задачи о его реберном покрытии. Кардинальное различие в сложности "вершинных" и "реберных" задач состоит в том, что первые относятся к разряду экспоненциально сложных, в то время как для вторых известны полиномиальные алгоритмы. Хорошо известен следующий результат.

Теорема 1 (Харари). Для каждого графа $G$ число вершин в любом вершинном покрытии $X$ не меньше числа ребер в произвольном паросочетании $M$, т.е. имеет место неравенство $|M| \leq|X| \mid$.

Поскольку для элементарной функции совместности вида (9) аксиома кратности выполняется $\left(a(v, w)=1 \rightarrow\left|\{v, w\} \cap F_{k}\right| \geq 1\right)$, то она выполняется и для любого паросочетания заданного графа $G$. Поэтому теорема 1 в качестве следствия дает следующее.

Свойство 8. Пусть для 1-подграфа тестирования $D_{1}=\left(V_{1}, E_{1}\right)$ его произвольное паросочетание имеет вид

$$
M\left(D_{1}\right)=\left\{(v, w): \quad v, w \in V_{1},(v, w) \in E_{1}\right\} .
$$


Удаление элементов паросочетания $M\left(D_{1}\right)$ из графа $D_{1}=\left(V_{1}, E_{1}\right)$ с корректировкой порога самоопределения на величину $\left|M\left(D_{1}\right)\right|$ применимо для условного самоопределения.

Преимущества, доставляемые использованием теоремы 1, состоят в следующем: 1) можно использовать построение конкретного паросочетания в качестве решения задачи о вершинном покрытии, при котором не нарушается аксиома кратности; 2) при использовании паросочетания для условной корректировки порога самоопределения из графа исключаются только и только элементы паросочетания, а оставшиеся элементы 1подграфа могут участвовать в дальнейшем диагностировании; 3) для построения наибольших паросочетаний известны многочисленные детерминированные и рандомизированные, точные и приближенные алгоритмы с полиномиальной сложностью как для отдельных классов графов, так и для графов общего вида, в том числе алгоритмы, допускающие параллельную децентрализованную реализацию. Лучший из известных алгоритмов построения наибольшего паросочетания имеет полиномиальную сложность $\mathrm{O}\left(\left|E_{1}\right| \sqrt{\left|V_{1}\right|}\right)$.

Кардинальным решением задачи о покрытии вершин 1-подграфа тестирования множеством неисправных вершин, при котором также автоматически обеспечивается соблюдение аксиомы кратности, является следующее.

С в о й с т в о 9. Если при выполнении очередного теста $(v, w)$ получено $a(v, w)=1$, то вериины, участвовавшие в выполнении этого теста, удаляются из диагностического графа вместе $c$ инциидентными им дугами $и$ с сопутствующим уменьшением на единицу порога самоопределения.

Использование такой оперативной условной корректировки порога самоопределения а) увеличивает вероятность продуктивности ранее образованных функций совместности и сопутствующее сокращение общего времени диагностирования, б) позволяет избежать образования больших 1-подграфов и применения трудоемких процедур их диагностического анализа, а для простых структур дает возможность быстрого определения числа паросочетания. Эти преимущества окупают возможную потерю эффективности диагностирования, вытекающую из соотношения $|M| \leq|X|$.

Описанное свойством 9 условное самоопределение вершин диагностического графа относится к вершинам, участвующим в выполнении теста, и называется прямым условным самоопределением. В работе [11] для ПМЧ-модели найдены правила косвенного условного самоопределения, при котором самоопределение пары вершин осуществляется на основе анализа исходов тестов над третьей вершиной.

Рассмотрим функции совместности для пар смежных тестов, образованных на множестве вершин $\{v, w, u\}$. Имеется три пары смежных тестов, различающиеся степенью полузахода / полуисхода соответствующих вершин: $\{(v, u),(w, u)\},\{(v, w),(w, u)\}$ и $\{(v, w),(v, u)\}$.

Анализ функций совместности, образованных для каждой из указанных пар смежных тестов, дал два следующих признака косвенного условного самоопределения.

Свойство 10. Если образ неисправностей $F_{k}$ порождает синдром $\sigma^{(r)}\left(F_{k}\right)$, такой, что для некоторой пары смежных тестов $\{(v, u),(w, u)\}$ выполняется

$$
\{a(v, u) \neq a(w, u)\} \subset \sigma^{(r)}\left(F_{k}\right),
$$

то при любом состоянии вершины и имеет место $\{v, w\} \cap F_{k} \neq \varnothing$.

Свойство11. Если образ неисправностей $F_{k}$ порождает синдром $\sigma^{(r)}\left(F_{k}\right)$, такой, что для некоторой пары смежных тестов $\{(v, w),(w, u)\}$ выполняется

$$
\{a(v, w)=0, a(w, u)=1\} \subset \sigma^{(r)}\left(F_{k}\right),
$$

$m o\{v, u\} \cap F_{k} \neq \varnothing$.

Для пары смежных тестов $\{(v, w),(v, u)\}$ отношения косвенного условного самоопределения не существуют.

При использовании косвенного условного самоопределения в случае свойства 10 вершины $\{v, w\} \quad$ с инцидентными им дугами из диагностического графа исключаются. В случае свойства 11 из диагностического графа исключаются вершины $\{v, u\}$ с инцидентными им дугами

Пусть $D_{T}(v)$ - подграф тестирования, индуцированный множеством вершин $\left\{v, R\left(\Gamma_{0}^{-1}(v)\right), R\left(\Gamma_{1}^{-1}(v)\right), R\left(\Gamma_{1}(v)\right)\right\} . \quad$ Обозначим $V_{1}(v)=v \cup R\left(\Gamma_{0}^{-1}(v)\right) \quad$ и $\quad V_{2}(v)=R\left(\Gamma_{1}^{-1}(v) \cup \Gamma_{1}(v)\right)$. Будем рассматривать полный двудольный граф с долями $V_{1}(v)$ и $V_{2}(v)$. Этот граф задает отношения между вершинами подграфа $D_{T}(v)$, соответствующие свойствам 10 и 11. Опираясь на свойства 10 и 11, в [11] доказано следующее.

Утверждение. K подграфу тестирования $D_{T}(v)$ применимо условное самоопределение, при котором из него удаляются вершины доли с 
меньшей мощчностью и равное число произвольно выбираемых вершин другой доли, а порог самоопределения уменьшается на величину $\left.\tau=\min \left\{\left|V_{1}(v)\right|, \mid V_{2}(v)\right) \mid\right\}$.

\section{9- Заключение}

Рассмотрен системный уровень самодиагностирования ВС при множественных отказах и исходах тестирования, соответствующих ПМЧ-модели.

Выведены условия достоверной идентификации технического состояния модуля $\mathrm{BC}$, производимой по результатам тестирования только из модулей, находящихся в его ограниченной окрестности. Такое самодиагностирование названо локальным $t$ диагностированием, а условия идентификации состояния модуля - условиями его самоопределения. Предложена классификация локальных условий идентификации состояния модулей ВС по характеру оценки исходов тестов, используемых для самоопределения состояния модулей. Описан избыточный набор достаточных условий самоопределения модулей, которые используются для определения фактического состояния модулей в ходе самодиагностирования. Применение этих условий исключает необходимость выполнения над системой всех возможных тестов. Впервые показана возможность самоопределения состояния модулей без необходимости выполнять непосредственный тест между ними.

Предложенные локальные условия самоопределения модулей могут использоваться для построения децентрализованных алгоритмов самодиагностирования масштабируемых живучих вычислительных систем. многопроцесорных

\section{References:}

1. Preparata FP, Metze G, Chien RT (1967) "On the connection assignment problem of diagnosable systems", IEEE Trans. Electr. Comput. № 12. pp. 848-854.

2. Dimitriev YK (1993) "Samodiagnostika modulnykh vychislitelnykh sistem", Novosibirsk: VO "Nauka" 293 p.

3. Dimitriev YK (1978) "Samodiagnostika sistem iz odnotipnykh blokov", V kn.: Voprosy teorii i postroyeniya vychislitelnykh sistem Novosibirsk № 3 pp. 107-121.

4. Dimitriev YK (2006) "Lokalnaya samodiagnostika vychislitelnykh sistem pri mnozhestvennykh otkazakh", Vestnik TGU, Prilozheniye № 17 pp. 198-202.

5. Dimitriev YK (2007) "Local diagnostication in computer systems with the circulant structure", Automation and Remote Control V. 68 № 3 pp. 545-556.

6. Dimitriev YK, Zadorozhny AF (2007) "Usloviya lokalnogo samodiagnostirovaniya $\mathrm{v}$ vychislitelnykh sistemakh s tsirkulyantnoy strukturoy", Vestnik TGU. Prilozheniye. №23. pp. 216-220.

7. Hakimi SL, Amin AJ (1974) "Characterization of connection assignement of diagnosable systems", IEEE Trans. on Comput. Vol. C-23. № 1. pp. 86-88.
8. Dimitriev YK (2010) "Analysis of the Possibility of Local Diagnosis in Computer Systems with Circulant Structure Based on Redundancy in the Number of Analyzed Testing Results" Automation and Remote Control Vol. 71, № 4 pp. 697-707.

9. Dimitriev YK (2008) "Partial and Complete Local Diagnosis in Computer Systems with Circulant Structure", Automation and Remote Control Vol. 69 № 7 pp. 1223-1230.

10. Dimitriev YK (2012) "Conditional local selfregulation rules and a diagnostic algorithm for a multiprocessor system with circulant diagnostic structure based on these rules", Automation and Remote Control Vol. 73 №5 pp. 862872.

11. Dimitriev YK (2015) "On the conceptual foundations of comparative analysis and solution of self-diagnostic problems in multiprocessor systems under different unreliable testing models", Automation and Remote Control Vol. 76 №7 pp. 1260-1270.

12. Listrovoy SV, Yablochkov SV (2003) "Metod resheniya zadachi opredeleniya minimalnykh vershinnykh pokrytiy i nezavisimykh maksimalnykh mnozhestv", Elektronnoe modelirovaniye. Vol. 25 № 2 pp. 31-43. 